Journal of Microbiological Methods (2012) Volume 88, Issue 3, Pages 413-416.

Comparison of different bead-beating RNA extraction strategies: an optimized method for

\title{
filamentous fungi
}

Gonçalo M. Leite, Naresh Magan, Ángel Medina*

Applied Mycology Group, Cranfield Health, Cranfield University, Cranfield, Bedfordshire.

MK43 OAL, U.K.

Running title: Rapid extraction of fungal RNA using bead-beating

\section{* Correspondent footnote:}

Dr. Angel Medina-Vaya

Applied Mycology Group, Cranfield Health,

Cranfield University,

Cranfield, Bedfordshire. MK43 OAL, U.K.

Telf: (+44)01234-758300 ext.8313

Fax: 01234-758380

Email: a.medinavaya@cranfield.ac.uk

\begin{abstract}
Filamentous fungi are responsible for a wide range of useful pharma and toxic secondary metabolite products. Functional genomic studies have been hindered in filamentous fungi by their robust cell wall and endogenous RNase activity. To further take advantage of their biotechnological capabilities an easy high throughput method for extraction of good quality RNA is required. We describe an effective, robust high throughput method to extract intact RNA from filamentous fungi. Two bead beating systems with different motion patterns and speed capacities were tested for the extraction protocol. They were evaluated based on the total RNA yield and overall RNA quality. The high speed bead beating with glass beads associated with an automated purification method gave more than three times higher yields of total RNA with less than a quarter of the amount of mycelium required, while still maintaining its integrity and overall quality, with RNA Quality Indicator (RQI) numbers consistently above 7.5. This method also reduced cross contamination risks and kept RNA handling to a minimum while still being capable of multiple sample process reducing the time required to obtain RNA from filamentous fungi.
\end{abstract}




\section{Introduction}

Rapid advances in molecular biology have promoted the use of molecular techniques in mycological studies. As an example, new research trends with mycotoxigenic fungi have integrated traditional ecological and physiological experiments with molecular data (gene expression and gene switching) to use a systems approach to try and provide better insights into the functional role of gene clusters involved in mycotoxin production and a better understanding of fungal behaviour, especially in relation to environmental stresses (Schmidt-Heydt et al., 2009; Schmidt-Heydt et al., 2010; Schmidt-Heydt et al., 2011).

Thus, the adequate isolation and purification of fungal RNA is a crucial step to ensure the successful application of these techniques. The structure of the fungal cell wall makes their disruption for nucleic acid extraction difficult. For this reason the use of standard RNA extraction methods developed for animal cells, bacteria or yeasts cannot be readily applied. The most common techniques for fungal nucleic acid extraction are the use of cell wall degrading enzymes (Einsele et al., 1997; Williamson et al., 2000) and a physical grinding treatment undertaken with either dry ice or liquid nitrogen (Loeffler et al., 2001; Griffin et al., 2002; Al-Samarrai and Schmid, 2002). For RNA extraction this last physical treatment is the most common methodology applied. It consists of freezing the mycelium with liquid nitrogen and grinding it with the aid of a mortar and a pestle (Schmidt-Heydt et al, 2007; Abdel-Hadi et al., 2010).

A number of studies have reported promising results for mechanical bead-beating extraction procedures to isolate fungal DNA (van Burik at al., 1998; Griffiths et al, 2006; de Boer et al, 2010), but no special attention has been paid to methods for the isolation of RNA from cultured mycelium in agar medium. The technique has several advantages over the traditional methods including reduction in time, multiple sample extraction and a reduction of the risk of cross contamination. There is also no need for liquid nitrogen, which is often problematic and hazardous, especially when a large number of samples need to be processed.

To our knowledge, there is no protocol for RNA extraction using any high speed beadbeating method. The aim of this work was to design and develop a bead beating protocol for the extraction of high-quality RNA samples from filamentous fungi. Thus different glass, zirconium oxide, tungsten carbide and stainless steel beads of various sizes were examined in the Precellys 24 homogenizer (Bertin Technologies, Montigny le Bretonneux, France) and in the Tissuelysser LT (Qiagen, UK) instruments. Subsequently, the results obtained were compared with those obtained using the traditional method.

\section{Materials and methods}

\subsection{Fungal species and isolates maintenance}

An Aspergillus flavus strain (NRRL 3357) was used in this study. The strain was maintained on Malt Extract Agar (MEA, Oxoid, UK) at $25 \stackrel{\circ}{\circ}$. The cultures were subculture on Yeast Extract Sucrose agar (YES) which is composed of $20 \mathrm{~g} / \mathrm{L}$ of yeast extract (Fluka, USA), $150 \mathrm{~g} / \mathrm{L}$ of sucrose (Fisher scientific, UK) and $15 \mathrm{~g} / \mathrm{L}$ agar (Oxoid, UK). To enable fungal biomass harvesting, a sterile cellophane overlay disc $(8.5 \mathrm{~cm})$ was placed aseptically on the surface of each $9 \mathrm{~cm}$ Petri plate prior to inoculation. 


\subsection{Inoculation, incubation and mycelium collection}

Spores from a 7 day culture were collected using a sterile loop and placed in a sterile $25 \mathrm{ml}$ Universal bottle containing $10 \mathrm{~mL}$ of RO water (Direct-Q 3 UV, Millipore, France) with $0.05 \%$ Tween 80 . The spore suspensions was counted using a haemocytometer and adjusted to $1 \times 10^{5}$ spores per ml. Petri plates with YES and cellophane overlays were centrally inoculated with $10 \mu \mathrm{l}$ of the spore suspension and incubated at 25ㅇ during 10 days. After the incubation period, the cellophane discs containing whole colonies were collected under sterile conditions, quickly frozen in liquid nitrogen and stored at $-80^{\circ} \mathrm{C}$ until RNA extraction.

\subsection{RNA extraction methods}

In order to improve the extraction methodology and to achieve a high efficiency and reduced variability in the total RNA yields, several automated and traditional methods were compared.

In the interest of ensuring that the RNA samples obtained were suitable for RT-qPCR experiments, the MIQE guidelines (Bustin et al., 2009) were followed. The samples were quantified and the absorbance ratios including the $A_{260} / A_{280}$ ratio were measured using the Picodrop ${ }^{\circledR}$ (Picodrop Limited, Saffron Walden, UK). Gel electrophoresis, and RNA quality indicator numbers (RQI) were obtained using the Experion ${ }^{\mathrm{TM}}$ system (Bio-Rad Laboratories Ltd., Hertfordshire, UK).

\subsubsection{Mortar and pestle method}

The traditional method of grinding the mycelium in a mortar with a pestle using liquid nitrogen is the most common technique to extract RNA samples from filamentous fungi. Thus a sample of between $0.65-1 \mathrm{~g}$ biomass was ground in liquid nitrogen to a fine powder using the mortar and pestle. $150 \mathrm{mg}$ of this powder was then transferred to a $2 \mathrm{ml}$ Safe-Lock tube (Eppendorf, Germany), and $750 \mu$ of TRIzol (Invitrogen, USA) or RLT buffer (Qiagen, UK) were added. After a quick vortex (10 seconds) the samples were frozen at -80 o $C$ until RNA purification. TRIzol ${ }^{\circledR}$ was initially used due to its recognised RNA stabilizer properties, later the RLT buffer supplemented with $10 \mu \mathrm{l} / \mathrm{ml}$ of $\beta$-mercaptoethanol was used so that an evaluation between both extraction buffers could be achieved.

\subsubsection{Bead beating method}

A sample of $150 \mathrm{mg}$ of frozen biomass was placed into a $2 \mathrm{ml}$ extraction tube containing beads. $750 \mu \mathrm{l}$ of TRIzol, which denatures proteins and inhibits endogenous RNase activity and thus improves RNA stability while still facilitating the cell wall disruption, was added. The lysing properties of the RLT buffer (provided with the RNeasy Plant Mini Kit (Qiagen, Germany) were also evaluated; TRIzol ${ }^{\oplus}$ was replaced with the same amount of RLT buffer supplemented with $\beta$-mercaptoethanol. After a quick vortex the tubes were frozen at $-80^{\circ} \mathrm{C}$ until required.

The extraction was carried out in two different bead beaters, the Tissuelyser LT (Qiagen, UK) which has a characteristic 2D vertical movement with up to 50 oscillations per second, and the Precellys 24 (Bertin Technologies, Montigny le Bretonneux, France) with 3D motion with up to 6500 RPM. The bead material evaluated included glass, zirconium oxide, tungsten carbide and stainless steel beads of various sizes (Table 1). In conjunction with the TissueLyser LT, all beads supplied by the manufacture were tested, even though smaller size 
beads are recommended for use with filamentous fungi. In the Precellys we also tried all available beads sizes, although we did not try stainless steel beads since zirconium oxide beads were hard enough to break the mycelium cell wall and the $2.8 \mathrm{~mm}$ sized beads could be compared to the $3 \mathrm{~mm}$ tungsten carbide beads since both are ceramic materials. Both systems were used at their maximum speed. Two types of procedure were used:

a) Stainless steel and tungsten carbide beads were used in conjunction with the Tissuelyser LT, with all samples agitated for 1 minute with a 10 second interval followed by another 1 minute agitation. During the interval and after the cycle, the samples were cooled down by placing them in ice.

b) The glass and zirconium oxide beads were used with the Precellys 24 . They were agitated for 25 seconds followed by a 5 second interval and another 25 seconds agitation. Afterwards samples were immediately cooled down by placing them in ice.

In both cases, the final mixture was centrifuged at $16000 \mathrm{~g}$ for $10 \mathrm{~min}$ at 4 으 $\mathrm{C}$ in a temperature controlled centrifuge. The supernatants were collected in a $2 \mathrm{ml}$ Safe-Lock tube (Eppendorf, Germany).

\subsubsection{Bead beating parameters optimization}

Different cycles were assessed in order to increase the total RNA extraction. Modifications consisted in increasing the beating time by up to 5 minutes, increasing the cooling in between the cycles for up to 5 minutes and increase of the beating cycles and adding cooling steps.

\subsection{Evaluation of variability and repeatability}

The precision was determined by repeatability (intra-day) and intermediate precision (interday).

Repeatability was evaluated by assaying 6 samples $(3+3)$ from the same biomass sample on the same day. Intermediate precision was studied by comparing 12 samples $(6+6)$ on different days. The intra-operator variability was evaluated by independent analyses of 12 replicates of the same sample on the same day by two different operators. Total RNA concentration relative standard deviations (RDS) were calculated.

\subsection{Total RNA purification and integrity evaluation}

The purification was carried by the QiaCube (Qiagen, Germany), using the RNeasy ${ }^{\circledast}$ Plant Mini Kit (Qiagen, Germany), with the appropriate protocol. The RNA concentration was determined spectrophotometrically using a $2.5 \mu \mathrm{l}$ aliquot on the Picodrop ${ }^{\mathrm{TM}}$, the $A_{260} / A_{280}$ ratio was also obtained for initial RNA purity assessment. To fully evaluate RNA integrity and quality, $1 \mu \mathrm{l}$ aliquot was used as described by the manufacturer on the Experion ${ }^{\mathrm{TM}}$ system using the RNA StdSens Analysis chip (Bio-Rad, UK). An electropherogram was generated by the Experion ${ }^{\mathrm{TM}}$ system for each sample from which the RNA Quality Indicator (RQI) was automatically calculated and a virtual gel could be generated.

\subsection{Statistical analysis}

Analyses were done using the JMP 8 Statistical Software package (SAS Institute Cary NC, USA). Generally, the independent variable distribution was assessed using the Shapiro-Wilk W Test and the homoscedasticity was tested using the Levene's test. When any of them failed, a variable transformation was performed in order to improve normality or 
homogenize the variances. Either non-parametric (Kruskal-Wallis rank sum test) or parametric ANOVA test were used depending on the dataset characteristics. Afterwards post-hoc Tukey-Kramer Honestly Significant Difference (HSD) test was applied to investigate the relationship between the different treatment averages.

\section{Results}

3.1. Comparison between the traditional and bead-beating methods.

An overall comparison of total RNA obtained from each $100 \mathrm{mg}$ of biomass extracted, for all methods tested, is shown in Figure 1. Data was analysed using the Kruskal-Wallis test and significant differences were found between the total RNA amounts isolated using the different bead treatments and the manual system as factors ( $p$-value $=0.0072$ ). Further analysis using the Tukey-Kramer HSD test revealed that the total RNA obtained using the manual method was statistically lower than the total RNA obtained using glass and zirconium beads (VK01, VK05, CK14 and CK28). Consequently, stainless steel and tungsten carbide beads were removed from the data set. The remaining data (including glass and zirconium beads and the manual method) were analysed by ANOVA. Results confirmed the statistically significant difference on the total RNA obtained with the different procedures ( $p$-value<0.0001). Additional post-hoc analysis confirmed that glass beads provided the best recovery yields. Among them VKO5 (glass beads of $0.5 \mathrm{~mm}$ diameter) resulted in the highest amount of total RNA. Statistical analysis did not show any differences with VKO1 but differences were found with respect to all the other treatments. Results of the average total RNA extracted per $100 \mathrm{mg}$ of biomass are shown in Figure 2.

In order to verify the general quality of the RNA, the $A_{260} / A_{280}$ ratio was measured in all the samples. Measurements were performed in water under neutral $\mathrm{pH}$ conditions using the Picodrop. Ratios obtained for the manual method as well as for the bead beating method were close to the threshold number of 2 and were considered satisfactory. Based on the overall results obtained and based on the higher total RNA yield, optimum absorbance ratios and low variability of the results, the VK05 glass beads were selected as the best for extraction of fungal RNA.

\subsection{Glass bead beating protocol optimization and quality parameters}

Further modifications were examined with the objective of increasing the total RNA extraction yield using the VK05 glass beads. In order to discriminate between treatments, different quality parameters and thresholds were used. As a first threshold of quality, trials in which the $A_{260} / A_{280}$ ratio were not in the range between 1.8 and 2.1 were rejected. It should be noted that the absorbance ratios only provides an indication of the RNA purity, so in order to ensure that the RNA obtained using the different bead beating protocols had conserved integrity and could be used for quantitative analysis, the Experion ${ }^{\mathrm{TM}}$ system was used to study the total RNA quality and integrity. As an example of our quality threshold a virtual gel generated from the electropherogram of samples extracted using different glass bead beating protocols, including 3 rejected and 3 accepted samples, are shown in Figure 3 . Lane's 1-3 show admissible absorbance ratios but which failed our integrity threshold. This shows that while a satisfactory amount of RNA was present, degradation was evident. This is reflected in the RQI numbers which were lower than 4.5. Only samples with electropherograms similar to lanes 4-6 were accepted. Good integrity of the two RNA subunits ( $18 \mathrm{~S}$ and $28 \mathrm{~S}$ ) can be observed and RQI values were always $>6.5$, a value that was 
adopted as a good quality threshold. Samples that displayed a RQI between 4.5 and 6.5 would require further analysis if they were to be used in RT-qPCR experiment.

Final comparisons were made between protocols with VK05 glass beads by extracting 12 replicates of the same fungal biomass sample. Six were extracted using the methodology described in Section 2.3.2b ( 25 secs +5 secs cooling +25 secs) and the other 6 by agitating for 15 secs followed by 5 mins cooling, 25 secs of agitation, followed by another 5 mins of cooling and a final cycle of agitation for 15 secs. The second method gave a total RNA final yield of $20.7 \%$ higher than the former. RQI numbers ranged between 9.7 and 6.5 in the shorter protocol described in Section 2.3.2b which included two beating cycles of 25 secs interrupted by a cooling step of 5 secs and from 3.0 to 6.3 in the protocol that included more cooling steps. Increasing the beating period lead to a rapid decrease in RNA integrity with most samples having complete degradation of the ribosomal RNA by increasing the beating period to $>2$ mins.

After comparison of RNA integrity RQI numbers the method described in Section 2.3.2b with only 50 secs total beating time in two different cycles with a $5 \mathrm{sec}$ interval to prevent overheating of the samples was selected as the optimum extraction protocol.

\subsection{Performance studies of the chosen method}

Intra- and inter-day and inter- and intra-operator variability were tested for the proposed method. Intra-day (6 replicates) and inter-day variability were $8.01 \%$ RSD and $9.24 \%$ RSD respectively, suggesting good consistency with this method.

The intra-operator variability after extraction of 12 samples (6 samples each) was $6.61 \%$ RSD. These data were analysed using the Student's statistical test to examine whether there was statistically significant differences between operators. The $p$-value was 0.66 , showing that no significant differences were found.

\section{Discussion}

The cell wall of filamentous fungi is composed of chitin, (1-3)- $\beta$-D-glucan, (1-6)- $\beta$-glucan, lipids and peptides which affects the ability to effectively extract DNA and RNA for genetic studies (Francesconi et al., 2008). This has often hindered the development of high throughput systems for the extraction of nucleic acids from filamentous fungi.

In this study, we have demonstrated a fast semi-automated high throughput bead-beating protocol for the extraction and purification of total RNA from filamentous fungi with high yields of total RNA while conserving the overall quality and integrity. We have addressed two major bottlenecks that have been found in the recovery of nucleic acids from filamentous fungi. The first was to effectively gain access to the interior of the cell. This step has a profound impact on the total RNA yield and quality. RNA structure and chemical instability allied with high content of endogenous RNase in filamentous fungi means that temperature and buffering conditions can significantly influence not only yield but also RNA integrity and overall quality.

Previous osmotic pressure methods cannot be readily applied as the cell wall of filamentous fungi impedes lysis (Fredricks et al., 2005). The use of enzymes for cell wall digestion is 
possible, nevertheless the conditions used for enzymatic digestion of the cell wall could result in rapid degradation of the RNA by endogenous RNase activity. This would also increase the already high level of protein contamination of the sample which would potentially affect the downstream applications. Faster and more aggressive methods have also been used for membrane disruption including sonication (van Burik et al. 1998), microwave radiation (Ferreira and Glass, 1996; Tendulkar et al., 2003) and thermal treatment (Liu et al., 2011).

All these methods would have a detrimental effect on RNA extraction. The thermal susceptibility of RNA would exclude thermal treatment as well as microwave radiation. Our data showed that an increase in temperature, caused by increasing the beating period without adjusting the cooling period, lead to dramatic decreases in RNA integrity and quality. Sonication alone has not proved to be very efficient with DNA and for this reason was also excluded from our study (van Burik et al. 1998). Most of these alternatives have only been used with DNA and there are no studies suggesting their effectiveness and efficiency in extracting RNA from filamentous fungi.

Physical grinding methods using the mortar and pestle or bead beating have been recommended to extract DNA, with the latter giving the best extraction efficiency with fungal samples (van Burik et al. 1998; Haugland et al., 2002; White et al., 2010). There are also some method variations which include not only physical grinding methods using the mortar and pestle with sonication (Schmidt-Heydt et al., 2009), but these methods are still very time consuming and still have disadvantages.

Although, recently several authors have demonstrated improved methods to extract nucleic acids, mainly DNA, for PCR screening of clinically important yeasts and moulds (Alshahni et al., 2009; Fredlund et al., 2008; Liu et al., 2011; Manzanilla-López et al., 2009; Zhang et al., 2010), the challenge for a fast high-throughput and reliable protocol that could be widely used in fungi has still not been achieved. Although some faster and easier methods have recently been developed (Liu et al., 2011), these have not been appropriate for more sensitive techniques such as $\mathrm{qPCR}$, where better quality extracted nucleic acids are required (Bustin et al., 2009), and have often been found to be unsuitable, due to the potential degradation of RNA during the initial cell wall disruption.

In the present study, the use of bead beating methods gave consistently high yields of good quality total RNA even though the high speed employed generates a temperature increase during the bead beating process which potentially could also lead to RNA degradation. To assess the influence of cooling steps would have on the RNA integrity. The samples were cooled in between each bead beating step for up to 5 minute intervals. During this interval the samples were cooled by placing them in ice or briefly in liquid nitrogen or even by stopping the bead beating and resuming it after a few seconds which led to a lower final temperature and to higher quality RNA.

Furthermore, increasing the time of the beating process resulted in a lowering of the total RNA quality. In fact, the attempts made to increase the yield by increasing the beating periods produced degradation of the RNA. This was probably due to a slight increase in temperature and the buffer being unable to completely inhibit the RNase activity. Thus, the 
RNA was degraded by endogenous RNases released from the fungal biomass. The second hurdle is how to separate the nucleic acid of interest from the highly complex mixture of proteins, polysaccharides and other mycelium debris resulting from the extraction procedure and still avoiding RNA degradation. In silica membrane column purification is a widely used purification method in many purification kits. However, contamination with polysaccharide can reduce their efficiency significantly (Gambino et al., 2008). Thus a prefilter column can be used to retain some contaminants and improve homogenization of the lysate. Thus we employed Qiagen's Qiashredder columns. These columns allowed an initial clean-up of the samples which lead to a higher purity of the final eluate. Initially we used TRIzol $^{\circledR}$ reagent during the lysis step, as it is a recognised RNA stabilizer while still holding lysing properties, facilitating cell disruption and dissolving cell components. TRIzol ${ }^{\circledR}$ reagent is a monophasic solution of phenol and guanidine isothiocyanate first described by Chomczynki and Sacchi (1987) that has been extensively used for the isolation of total RNA from cells and tissues with good results.

TRIzol $^{\circledR}$ reagent was later compared with the lysing buffer provided with the RNeasy Plant mini kit, the RLT buffer. Wang et al. (2008) showed an increase in RNA quality and in some applications RNA yield by using TRIzol ${ }^{\circledR}$ reagent in conjunction with the Qiagen RNeasy Mini Plant kit. In our studies, on the other hand the RLT lysis buffer gave similar quality total RNA with a slight increase in yield, which might be due, to some extent, to the improved lysing effect on the ground fungal biomass during the bead beating process. The RLT buffer is based on guanidine thiocyanate salts. This buffer was supplemented with $\beta$ mercaptoethanol which irreversibly denatures RNases. To improve the RNA quality, polyvinylpyrrolidone (PVP) which can inhibit the polyphenol oxidase of nucleic acids that result on their degradation can also be used (Chan et al., 2007; A. Abdel-Hadi et al., 2010).

This second step could still be further improved by using cationic surfactants like cetyl trimethylammonium bromide (CTAB) which could still provide a slight improvement on the total RNA yield as suggested by Wang and Stegemann (2010). However, they were extracting RNA from human mesenchymal stem cells from rich polysaccharide matrices. Nevertheless, the low yield of DNA achieved with Fusarium species would suggest that it might not improve the total RNA yield from filamentous fungi (Fredlund et al., 2008). This method would also be too laborious to be considered as a high throughput system.

Fredlund et al. (2008) reported more consistency in DNA yield when extracting from Fusarium species, when using an automatic robotized method. The use of an automated method for purification allows a decrease of sample handling time, less chance of cross contamination risk and reduced variability. This enables a comparison to be made between the different extraction methods and conditions during RNA purification. We used the QiaCube (Qiagen) automated platform in our studies.

This showed that it was possible to develop a fast and reliable method to produce high quality RNA that can be subject to functional genomic studies by RT-qPCR. We followed the MIQE guidelines (Bustin et al., 2009) while developing this method. This is important for generating significant and relevant data from RT-qPCR experiments. Indeed, the first step to generate good reliable data is to be able to extract high quality nucleic acids.

In developing a systems approach linking molecular, ecology and physiology data, a high throughput system is essential to generate the necessary data. With the method developed 
in this study it will be possible to process a large number of samples in a short period of time ( $x$ samples per $8 \mathrm{hr}$ day) which will contain higher total RNA yields and overall quality than when using the traditional method.

Even though our reported protocol focuses on high quality RNA, it could potentially be adapted to DNA extraction and help in the large scale genomic studies in filamentous fungi to take fully advantage of their biotechnology capabilities (Meyer, 2008). This method has been used at Cranfield for several mycotoxigenic fungal species (i.e. F. langsethiae, $F$. verticillioides, $F$. graminearum and $P$. verrucosum) grown in both laboratory media and on grain matrices with similar (data not shown).

\section{References}

Abdel-Hadi, A., Carter, D., Magan, N., 2010. Temporal monitoring of the nor-1 (aflD) gene of Aspergillus flavus in relation to aflatoxin B1 production during storage of peanuts under different water activity levels. J. Appl. Microbiol. 109, 1914-1922.

Al-Samarrai, T.H., Schmid, J., 2000. A simple method for extraction of fungal genomic DNA. Lett. Appl. Microbiol. 30, 53-56.

Alshahni, M.M., Makimura, K., Yamada, T., Satoh, K., Ishihara, Y., Takatori, K., Sawada, T., 2009. Direct colony PCR of several medically important fungi using Ampdirect ${ }^{\circledR}$ plus. Jpn. J. Infect. Dis. 62, 164-167.

Bustin, S.A., Benes, V., Garson, J.A., Hellemans, J., Huggett, J., Kubista, M., Mueller, R., Nolan,T., Pfaffl, M.W., Shipley, G.L., Vandesompele, J., Wittwer, C.T., 2009. The MIQE Guidelines-Minimum Information for Publication of Quantitative Real-Time PCR Experiments. Clin. Chem. 55, 611-622.

Chan, K.L., Ho, C.L., Namasivayam, P., Napis, S., 2007. A simple and rapid method for RNA isolation from plant tissues with high phenolic compounds and polysaccharides. Nat. protoc. doi:10.1038/nprot.2007.184.

Chomczynski, P., Sacchi, N., 1987. Single-step method of RNA isolation by acid guanidinium thiocyanate-phenol-chloroform extraction. Anal. Biochem. 162, 156-159.

De Boer, R., Peters, R., Gierveld, S., Schuurman, T., Kooistra-Smid, M., Savelkoul, P., 2010. Improved detection of microbial DNA after bead-beating before DNA isolation. J. Microbiol. Methods 80, 209-211.

Einsele, H., Hebart, H., Roller, G., Loffler, J., Rothenhofer, I., Muller, C.A., Bowden, R.A., Van Burik, J.-A.H., Engelhard, D., Kanz, L., Schumacher, U., 1997. Detection and identification of fungal pathogens in blood by using molecular probes. J. Clin. Microbiol. 35, 1353-1360.

Ferreira, A.V.B., Glass, N.L., 1996. PCR from fungal spores after microwave treatment. Fungal Genet. Newsl., 43, 25-26.

Francesconi, A., Kasai, M., Harrington, S.M., Beveridge, M.G., Petraitiene, R., Petraitis, V., Schaufele, R.L., Walsh, T.J., 2008. Automated and manual methods of DNA extraction for Aspergillus fumigatus and Rhizopus oryzae analyzed by quantitative real-time PCR. J. Clin. Microbiol. 46, 1978-1984.

Fredlund, E., Gidlund, A., Olsen, M., Börjesson, T., Spliid, N.H.H., Simonsson, M., 2008. Method evaluation of Fusarium DNA extraction from mycelia and wheat for down-stream real-time PCR quantification and correlation to mycotoxin levels. J. Microbiol. Methods 73, 33-40.

Fredricks, D.N., Smith, C., Meier, A., 2005. Comparison of six DNA extraction methods for recovery of fungal DNA as assessed by quantitative PCR. J. Clin. Microbiol. 43, 5122-5128. 
Gambino, G., Perrone, I., Gribaudo, I.A., 2008. Rapid and effective method for RNA extraction from different tissues of grapevine and other woody plants. Phytochem. Anal. 19, 520-525.

Griffin, D.W., Kellogg, C.A., Peak, K.K., Shinn, E.A., 2002. A rapid and efficient assay for extracting DNA from fungi. Lett. Appl. Microbiol. 34, 210-214.

Griffiths, L.J., Anyim, M., Doffman, S.R., Wilks, M., Millar, M.R., Agrawal, S.G., 2006. Comparison of DNA extraction methods for Aspergillus fumigatus using real-time PCR. J. Med. Microbiol. 55, 1187-1191.

Haugland, R.A., Brinkman, N., Vesper, S.J., 2002. Evaluation of rapid DNA extraction methods for the quantitative detection of fungi using real-time PCR analysis. J. Microbiol. Methods 50, 319-323.

Liu, K.-H., Yeh, Y.-L., Shen, W.-C., 2011. Fast preparation of fungal DNA for PCR screening. J. Microbiol. Methods, 85, 170-172.

Loeffler, J., Hebart, H., Cox, P., Flues, N., Schumacher, U., Einsele, H., 2001. Nucleic acid sequence-based amplification of Aspergillus RNA in blood samples. J. Clin. Microbiol. 39, 1626-1629.

Manzanilla-López, R.H., Clark, I.M., Atkins, S.D., Hirsch, P.R., Kerry, B.R., 2009. Rapid and reliable DNA extraction and PCR fingerprinting methods to discriminate multiple biotypes of the nematophagous fungus Pochonia chlamydosporia isolated from plant rhizospheres. Lett. Appl. Microbiol. 48, 71-76.

Meyer, V., 2008. Genetic engineering of filamentous fungi - Progress, obstacles and future trends. Biotecnol. Adv. 26, 177-185.

Schmidt-Heydt, M., Abdel-Hadi, A., Magan, N., Geisen, R., 2009. Complex regulation of the aflatoxin biosynthesis gene cluster of Aspergillus flavus in relation to various combinations of water activity and temperature. Int. J. Food Microbiol. 135, 231-237.

Schmidt-Heydt, M., Baxter, E., Geisen, R., Magan, N., 2007. Physiological relationship between food preservatives, environmental factors, ochratoxin and otapksPV gene expression by Penicillium verrucosum. Int. J. Food Microbiol. 119, 277-283.

Schmidt-Heydt, M., Rüfer, C.E., Abdel-Hadi, A., Magan, N., Geisen, R., 2010. The production of aflatoxin B1 or $\mathrm{G} 1$ by Aspergillus parasiticus at various combinations of temperature and water activity is related to the ratio of aflS to aflR expression. Mycotoxin Res. 26, 241-246.

Schmidt-Heydt, M., Parra, R., Geisen, R., Magan, N., 2011. Modelling the relationship between environmental factors, transcriptional genes and deoxynivalenol mycotoxin production by two Fusarium species. J. R. Soc. Interface. 8, 117-120.

Van Burik, J.-A.H., Schreckhise, R.W., White, T.C., Bowden, R.A., Myerson, D., 1998. Comparison of six extraction techniques for isolation of DNA from filamentous fungi. Medical Mycol. 36, 299-303.

Wang, C., Hao, J., Zhang, F., Su, K., Wang, D.-A., 2008. RNA extraction from polysaccharidebased cell-laden hydrogel scaffolds. Anal. Biochem. 380, 333-334.

Wang, L., Stegemann, J.P., 2010. Extraction of high quality RNA from polysaccharide matrices using cetlytrimethylammonium bromide. Biomater. 31, 1612-1618.

Williamson, E.C., Leeming, J.P., Palmer, H.M., Steward, C.G., Warnock, D., Marks, D.I., Millar, M.R., 2000. Diagnosis of invasive aspergillosis in bone marrow transplant recipients by polymerase chain reaction. Br. J. Haematol. 108, 132-139.

White, P.L., Bretagne, S., Klingspor, L., Melchers, W.J.G., McCulloch, E., Schulz, B., Finnstrom, N., Mengoli, C., Barnes, R.A., Donnelly, J.P., Loeffler, J., 2010. Aspergillus PCR: one step closer to standardization. J. Clin. Microbiol. 48, 1231-1240. 
Tendulkar, S.R., Gupta, A., Chattoo, B.B., 2003. A simple protocol for isolation of fungal DNA. Biotecnol. Lett. 25, 1941-1944.

Zhang, Y.J., Zhang, S., Liu, X.Z., Wen, H.A., Wang, M., 2010. A simple method of genomic DNA extraction suitable for analysis of bulk fungal strains. Lett. App. Microbiol. 51, 114-118. 


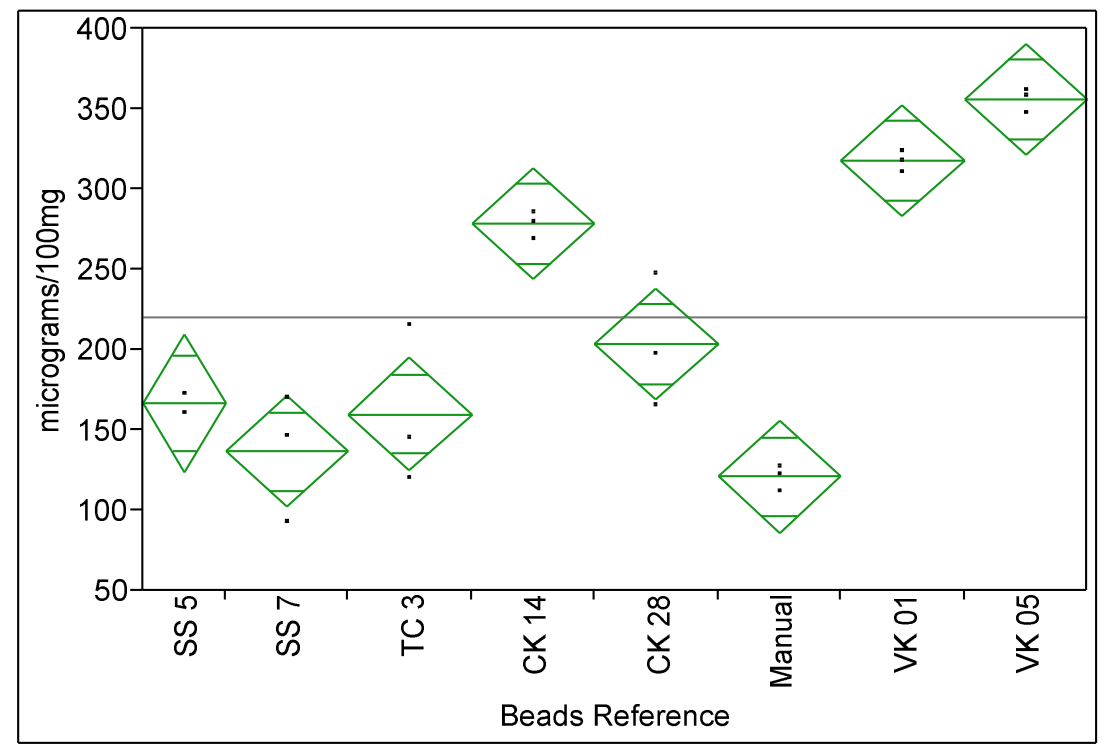

Figure $1 \quad$ Leite et al.

Figure 1: Diamond diagram obtained during Kruskal-Wallis test analysis of OD values/100mg of biomass using the different beads as factors ( $p$-value $=0.0072$ ). The line across each diamond represents the group mean. The vertical span of each diamond represents the $95 \%$ confidence interval for each group. Key to treatments: SS - Stainless Steel, TC - Tugsten Carbide, CK - Zirconnium Oxide, VK - Glass; followed by the bead size code $01-0.1 \mathrm{~mm}, 05$ $-0.5 \mathrm{~mm}, 14-1.4 \mathrm{~mm}, \mathbf{2 8}-2.8 \mathrm{~mm}, \mathbf{3}-3.0 \mathrm{~mm}, 5-5.0 \mathrm{~mm}$ and $7-7.0 \mathrm{~mm}$. 


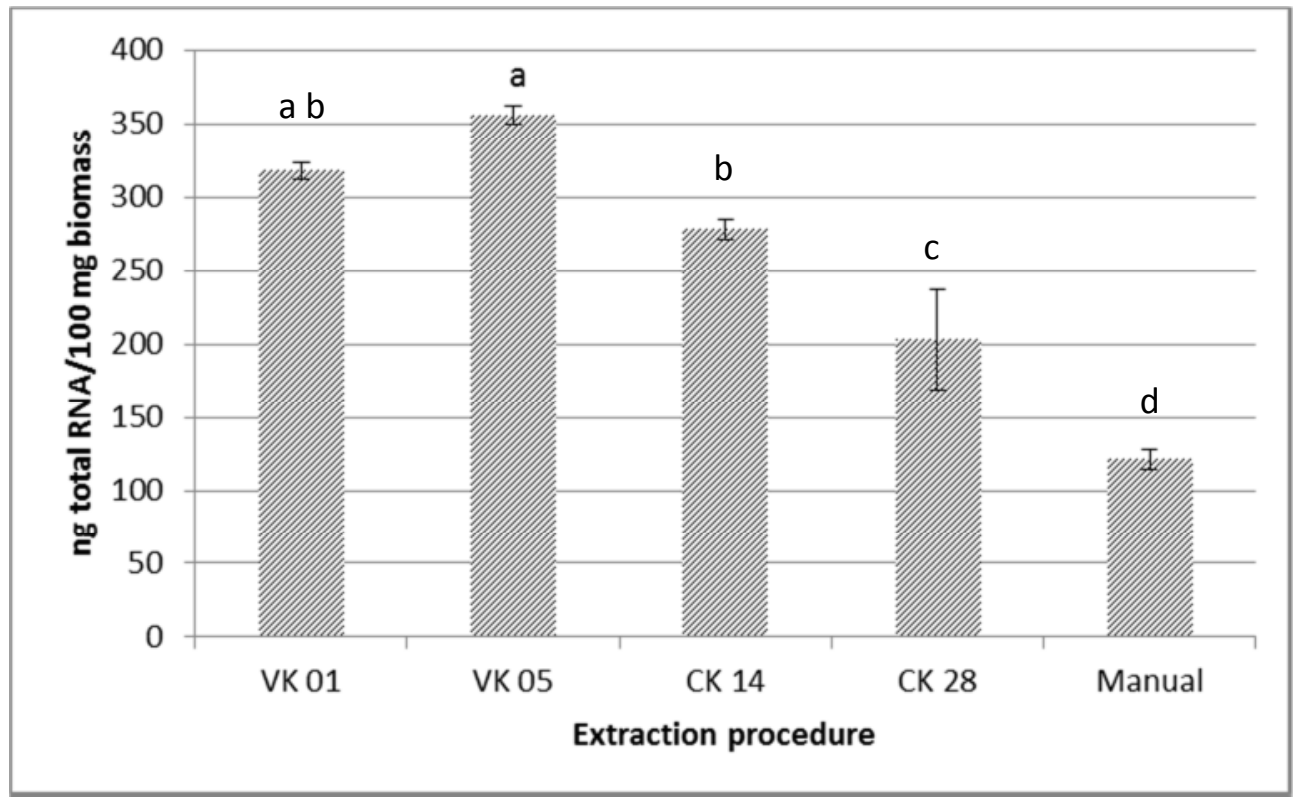

Figure 2: Total RNA yield average per $100 \mathrm{mg}$ of initial biomass and standard deviation comparing different beads with the manual method. Groups connected by different letters represent statistically different groups using Tukey-Kramer HSD test ( $p$-value $<0.0001$ ). Key to treatments: CK - Zirconnium Oxide, VK - Glass; followed by the bead size code $01-0.1$ $\mathrm{mm}, 05-0.5 \mathrm{~mm}, 14-1.4 \mathrm{~mm}, 28-2.8 \mathrm{~mm}$. 


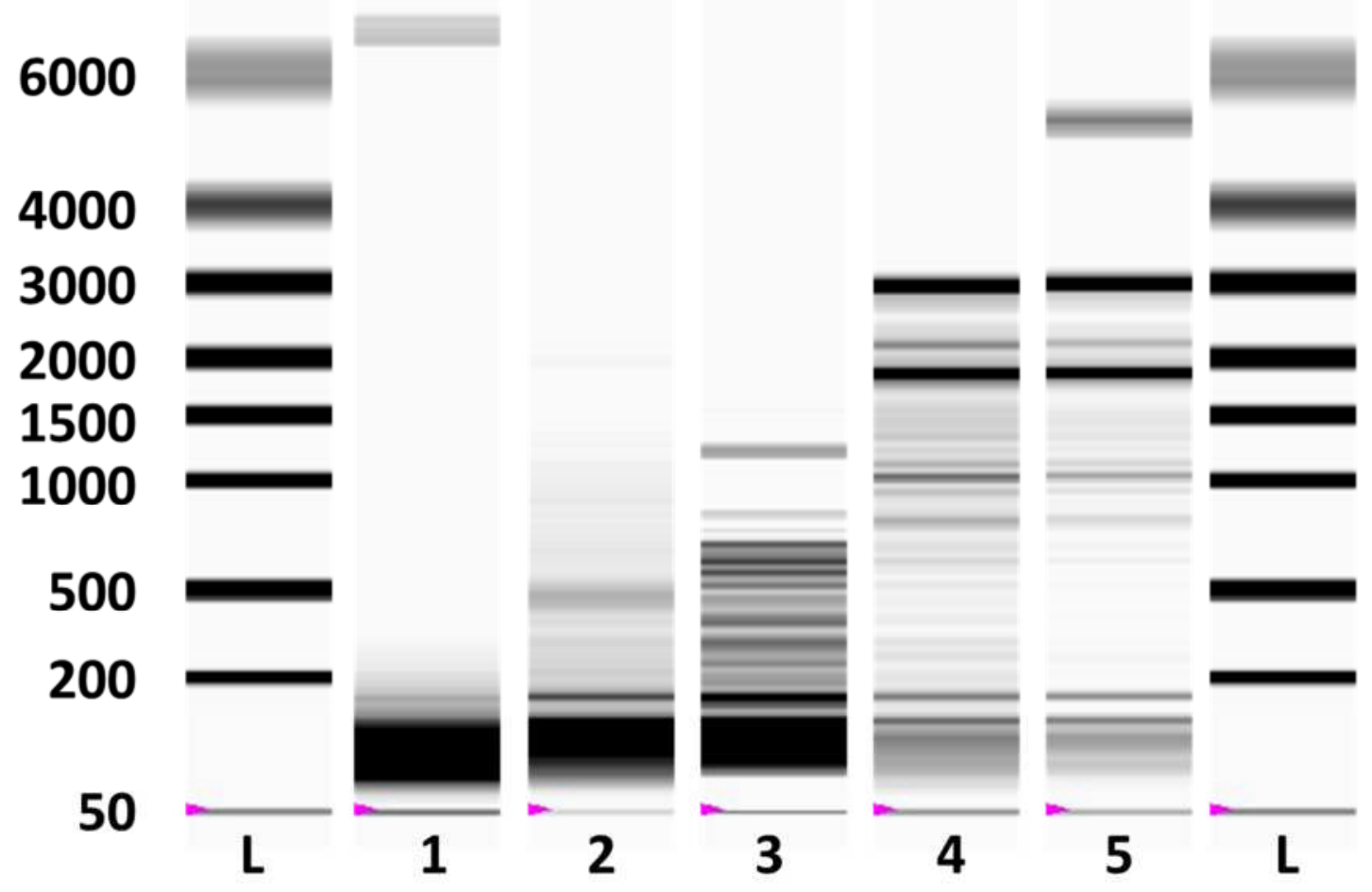

Figure 3: Virtual gel generated by BioRad Experion for samples of different quality (1-5). Lane 1 appears to possess un-denatured RNA, Lane 2 and 3 highly degraded RNA and Lane 4 and 5 have a RQI above 6.5 (Good Quality). Lane 5 shows some genomic DNA contamination. 


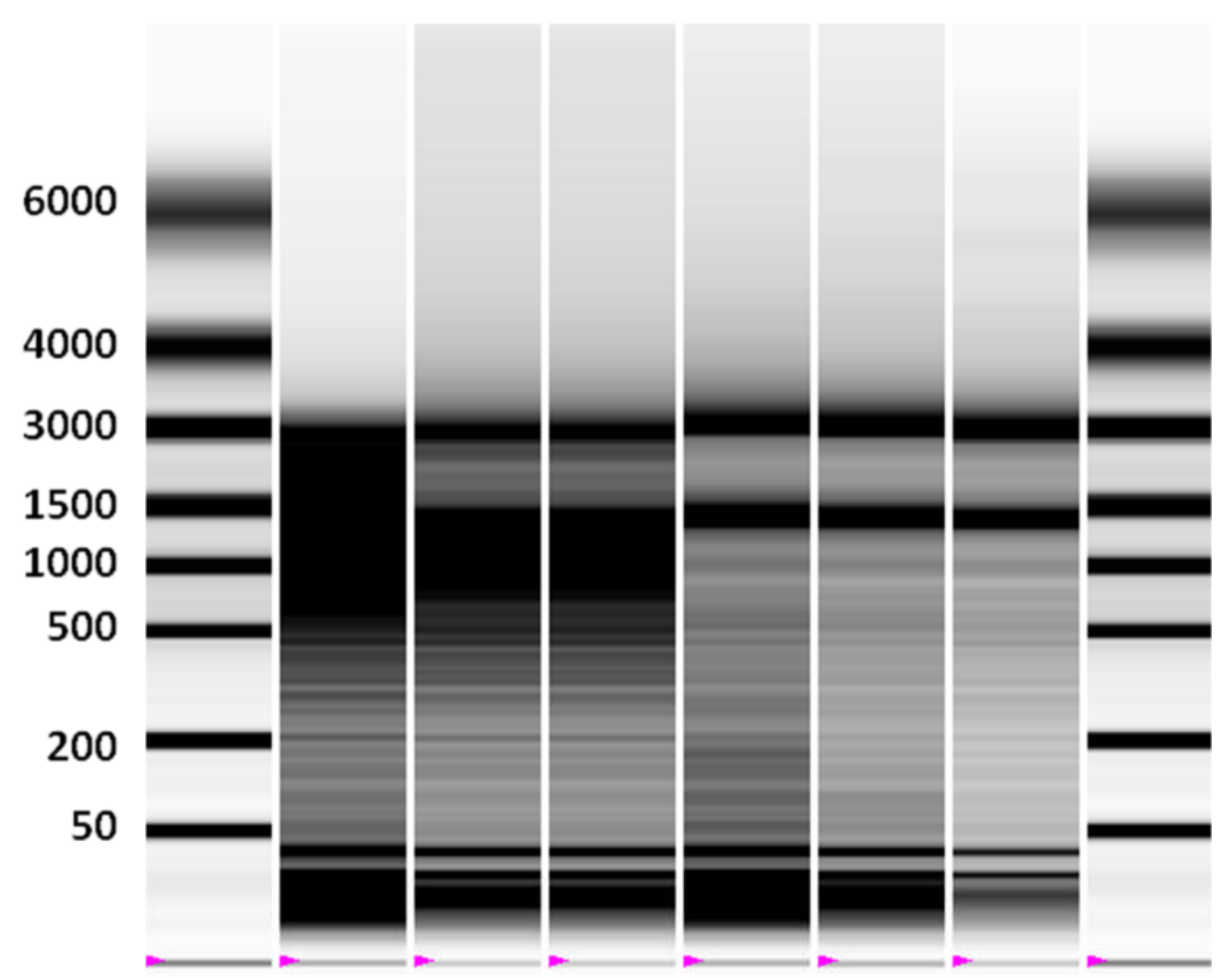

Figure 4: Virtual gel generated by BioRad Experion of samples extracted using different bead beating protocols. Lane 1, 2 and 3 samples were subjected to a 5 minute ice cooling step in between bead beating steps, Lane 4, 5 and 6 samples that were only subjected to a 5 second interval at room temperature between bead beating steps. The RQI of Lane 1 is 3.0, Lane 2 is 3.7, Lane 3 is 4.1 , Lane 4 is 6.5 , Lane 5 is 6.7 and Lane 6 is 7.7 on a $0-10$ scale. 


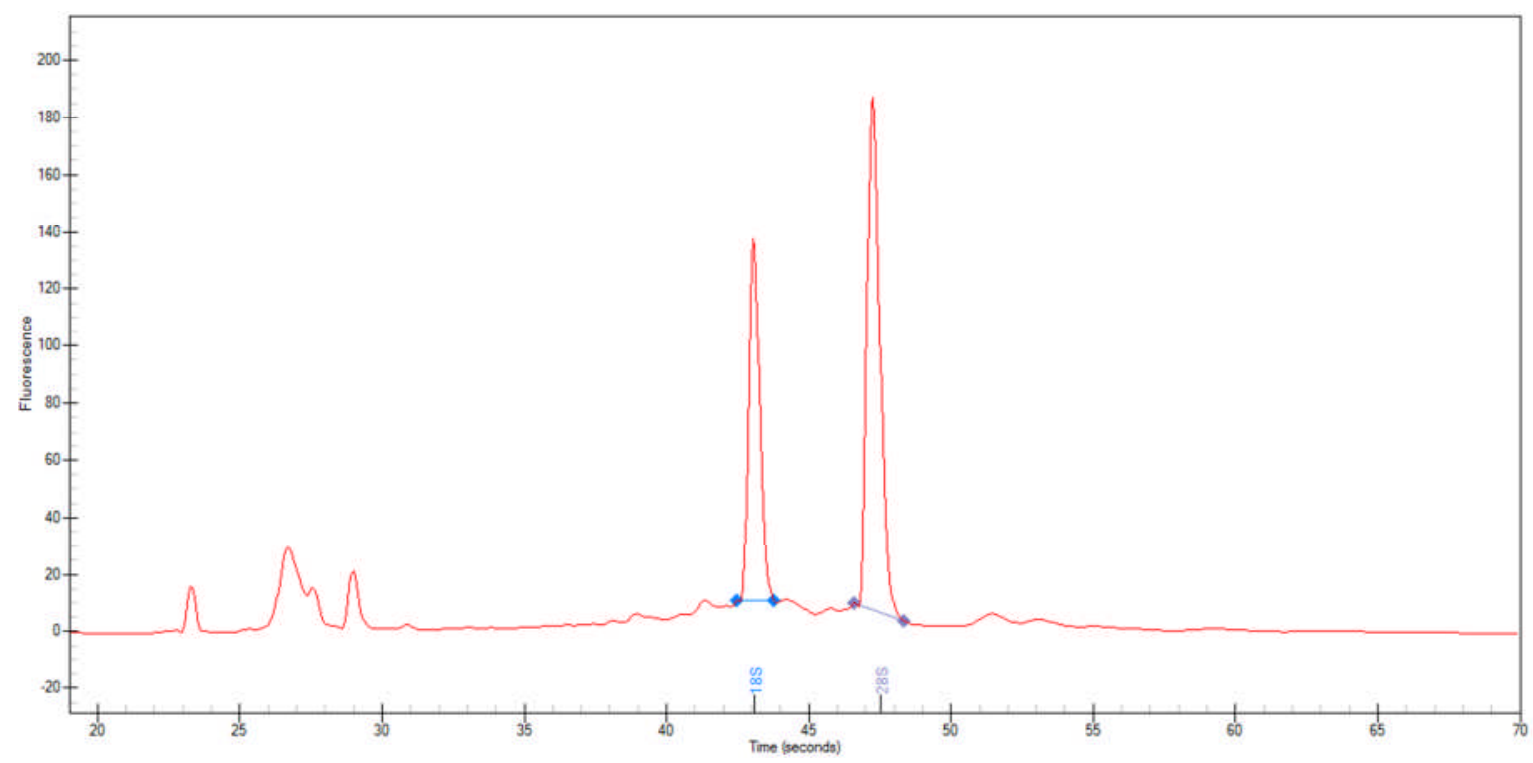

Figure 5. Leite et al.

Figure 5: Eletropherogram of a good quality RNA sample extracted using glass beads with two 25 seconds beating periods paused by only 5 seconds at room temperature. The RQI of this sample is 9.7.

Table 1. Summary of the beads characteristics including: reference, material and size used in these studies.

\begin{tabular}{ccc}
\hline Reference & Material & Size (mm) \\
\hline VK 01 & Glass & 0.1 \\
VK 05 & & 0.5 \\
\hline CK 14 & \multirow{2}{*}{ Zirconium Oxide } & 1.4 \\
CK 28 & & 2.8 \\
\hline TC 3 & Tungsten Carbide & 3.0 \\
\hline SS 5 & Stainless Steel & 5.0 \\
SS 7 & & 7.0 \\
\hline
\end{tabular}

